\title{
Approaches to Dealing with High-Sized Polynuclear Systems with ab initio Methods
}

\author{
Carmen J. Calzado ${ }^{1}$, Jean-Paul Malrieu ${ }^{2}$ and Daniel Maynau ${ }^{2}$ \\ 1. Departamento de Quimica Física. Universidad de Sevilla.E-41012 Sevilla. Spain.calzado@us.es \\ 2. Laboratoire de Chimie et Physique et Quantiques. IRSAMC. Université Paul Sabatier. F-31062 Toulouse. \\ France.malrieu@irsamc.ups-tlse.fr, daniel.maynau@irsamc.ups-tlse.fr
}

\begin{abstract}
This contribution summarizes some of the theoretical strategies developed in the recent past to study systems with an increasing size and complexity by means of accurate extended CI calculations. Some examples of the capabilities of these strategies are also given, concerning the evaluation of magnetic exchange constants, as well as many other effective interaction parameters, such as hopping integrals, on-site Coulomb repulsion, four-body exchange term, ... on 2D and spin-ladder cuprates, polyoxovanadates, as well as polynuclear molecular compounds.
\end{abstract}

Keywords: magnetic interactions, charge transfer, extended CI calculations, local orbitals, high-Tc cuprates, molecular magnets

PACS: 71.15.-n, 74.72.-h, 75.30.Et, 75.50.Xx

In recent years, there has been considerable interest in the theoretical study of the magnetic properties of both molecular and solid state magnets. Part of this interest has focused on the evaluation of magnetic exchange couplings in order to clarify and get a deeper understanding of the mechanisms controlling the magnetic interactions.

However, this is not a simple task for theoretical approaches, essentially due to the size and complexity of the systems showing these properties. A general characteristic of these compounds is the presence of several unpaired electrons, distributed in some active orbitals, usually quasi-degenerated. The so-resulting ground state wave function has an intrinsic multireference nature, which is at the origin of the failure of density functional theory based methods (DFT) in describing these highly correlated systems. In spite of that, some pragmatic solutions have been proposed, such as the combination of the broken-symmetry approach proposed by Noodleman with the use of hybrid functionals, which has provided J estimates in reasonable agreement with the experimental values.

On the other hand, ab initio extended Configuration Interaction (CI) calculations are inherently adapted to describe these systems. However they experience a main difficulty: the resources and computational time required are so large that could just frustrate their use, even when embedded small building blocks are employed in the calculations. To overcome these limitations, different ab initio approaches have been proposed in the past. Among them, the Dedicated Difference CI (DDCI) method [1] has been extensively used to study transition metal compounds, in particular, to evaluate energy differences. The procedure gives a criterion, based on perturbative arguments, to rationally select the configurations included in the CI expansion. This considerably reduces the size of the CI expansions, but without lost of accuracy in the evaluation of spectral energy differences. Quite good agreement with experimental data has been obtained in the evaluation of magnetic coupling constants and hopping integrals in both solid and molecular systems. Moreover, the method has resulted to be a quite valuable tool in the analysis of the main physical factors controlling the magnetic exchange [2-3] and the electron coupling constant [4].

Some examples of the application of this methodology will be shown, regarding the determination of magnetic exchange couplings, four-body exchange terms, hopping integrals, on-site Coulomb repulsion, ... for $2 \mathrm{D}$ and spinladder cuprates, as well as polyoxovanadates. A sample is collected in Table I, which reports on estimates of the first-neighbor magnetic coupling (J) for the $\mathrm{La}_{2} \mathrm{CuO}_{4}$ compound (Figure 1), parents for the high- $\mathrm{T}_{\mathrm{C}}$ superconductors. The experimental $J$ value for this system has been established from neutron scattering $(\mathrm{J}=-134 \pm 5 \mathrm{meV})[5]$ and

CP963, Vol. 2 Part A, Computation in Modern Science and Engineering, Proceedings of the International Conference on Computational Methods in Science and Engineering 2007, edited by T. E. Simos and G. Maroulis (C) 2007 American Institute of Physics 978-0-7354-0478-6/07/\$23.00 
Raman measurements ( $\mathrm{J}=-128 \pm 6 \mathrm{meV}$ ) [6]. DDCI calculations on embedded finite clusters give values quite close to the experimental one. The calculations on two- $\left(\mathrm{Cu}_{2} \mathrm{O}_{7}\right)$ and four-center $\left(\mathrm{Cu}_{4} \mathrm{O}_{12}\right)$ clusters allow us to rule out the dependence on the cluster size. Estimates obtained with DFT calculations are also included for the sake of comparison. B3LYP as well as most of the available correlation-exchange functionals supply incorrect $\mathbf{J}$ values. Only when a certain degree of mixing with Fock exchange is introduced in the functional, it is possible to recover the experimental value. Previous calculations have shown that the best percentage, at least for $\mathrm{NiO}$ [7] and cuprates, is around 35\% Fock (Fock-35 in Table I) [2,8]. Also the adequacy of the finite model used to represent the solid is checked, by comparing the values resulting from finite embedded cluster DFT calculations with those provided by periodic DFT ones [9].

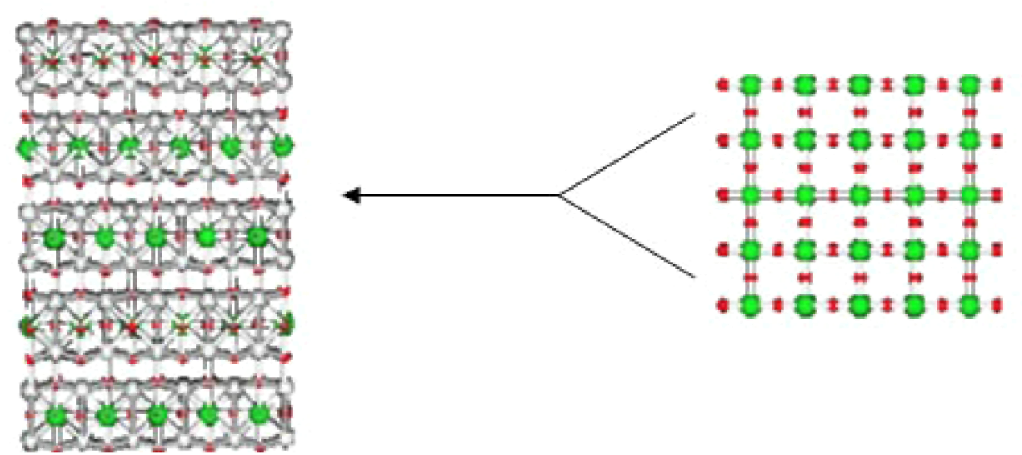

FIGURE 1. Crystal structure of $\mathrm{La}_{2} \mathrm{CuO}_{4}$ (left). A fragment of one of the two-dimensional $\mathrm{CuO}_{2}$ planes (right). Small white, large grey and small grey balls represent $\mathrm{La}, \mathrm{Cu}$ and $\mathrm{O}$ atoms, respectively.

The combination of the DDCI methodology with local orbitals has opened the use of this approach to larger systems, containing extended ligands as such as shown in Figure 2. Taking benefit of the locality of these MOs it is possible to freeze those of them placed far away from the active centers. This truncation considerably reduces the size of the inactive space and makes feasible calculations otherwise impractical. In particular for the binuclear $\mathrm{Cu}(\mathrm{II})$ compound shown in Figure 2, it is possible to recover $95 \%$ of the magnetic coupling value with only $10 \%$ of the total CI space, pointing to the potentiality of this strategy [10]. An alternative tool is the design of symmetry-adapted MOs especially dedicated to the evaluation of energy differences [11]. They are the eigenvectors of the difference of the density matrices of the involved states, which eigenvalues represent the degree of implication in the description of the energy difference. Similarly to the local orbitals, these dedicated MOs furnish also a truncation criterion of both the occupied and virtual inactive sets, and consequently of the CI space. Figure 2 shows the effect of the truncation of the DDCI space on the value of the J magnetic coupling in this binuclear $\mathrm{Cu}$ (II) compound, when dedicated MOs are used. When the complete MOs set is considered, the DDCI calculation recovers the experimental $\mathrm{J}$ value for this system. Eliminating in a progressive way those orbitals less implicated in the exchange coupling (with lower participation number), it is possible to reproduce $90 \%$ of the $\mathrm{J}$ value with only a $5 \%$ of the original CI space.
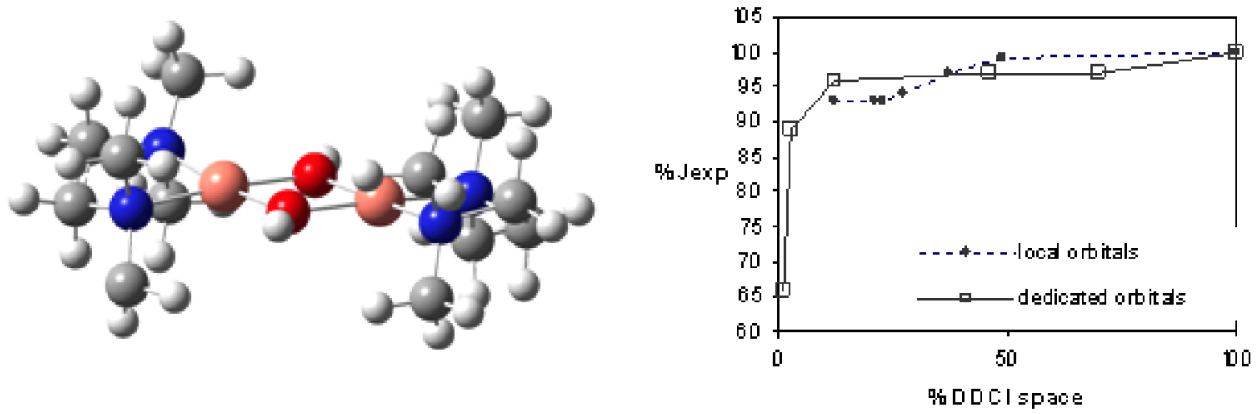

FIGURE 2. Structure of the binuclear $\mathrm{Cu}(\mathrm{II})$ compound $[\mathrm{Cu}(\text { tmeen }) \mathrm{OH}]_{2} \mathrm{Br}_{2}$, tmeen =tetramethylethylenediamine, which presents an antiferromagnetic coupling of $-509 \mathrm{~cm}^{-1}$ (Ref. 12). On the right, the effect of the inactive space truncation on the $J$ value, when local orbitals (open squares) or dedicated MOs (filled circles) are used. 
Although these strategies have demonstrated to be quite efficient to treat small systems and highly symmetric medium-sized systems, their application to high-sized systems is still a challenge. In this direction, it has been recently proposed a new criterion of truncation of the CI spaces, specially conceived for large systems [13]. It takes benefit of the use of local orbitals and consists in keeping only those excitations among "interacting" local orbitals. The strength of the interaction is measured by means of the exchange integral $\mathrm{K}_{\mathrm{ij}}=\langle i j \mid j i\rangle$. A $\mathrm{K}_{\mathrm{ij}}$ value larger than a threshold means that the $i$ and $j$ orbitals are "linked" and then the excitations involving them are kept on the CI space. Usually "linked" orbitals are also close in space. So, the method could be especially relevant for systems with a large spatial extension. An example where DDCI calculations are practicable by using this strategy is the evaluation of magnetic coupling constants for the ferromagnetic $\mathrm{Cu}_{4} \mathrm{O}_{4}$ cubane-like compound shown in Figure 3 . Indeed, in this particular case, the extracted $\mathbf{J}$ values serve to clarify the experimental assignments, in disagreement with the $\mathrm{Cu}-\mathrm{Cu}$ distances and relative orientation [14].

Finally, the advantages and limitations of the different strategies will be discussed, as well as possible extensions.
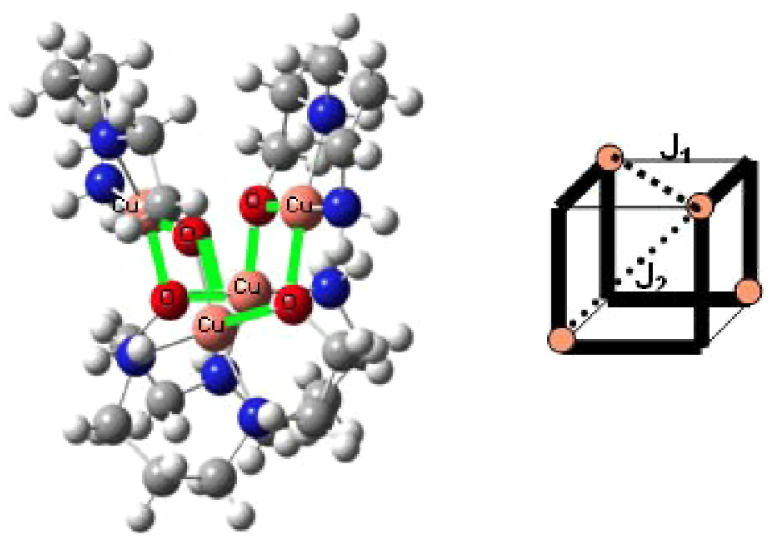

FIGURE 3. Structure of the cubane $\left[\mathrm{Cu}_{4}(\mathrm{hpda})_{4}\right]\left[\mathrm{ClO}_{4}\right]_{4} \cdot \mathrm{H}_{2} \mathrm{O}$, hpda= N-(2-hydroxi-etil)-1,3-propanodiamine (Ref. 15) and simplified model of the main magnetic interactions in this system.

TABLE 1. Magnetic coupling constant $\mathrm{J}$ (in meV) evaluated for $\mathrm{La}_{2} \mathrm{CuO}_{4}$ from finite cluster and periodic DFT calculations. Experimental J value: $-134 \pm 5 \mathrm{meV}[5],-128 \pm 6 \mathrm{meV}[6]$.

\begin{tabular}{cccc}
\hline model & DDCI & B3LYP & Fock-35 \\
\hline finite two-center cluster & $-125[15]$ & $-209[2],-237[18]^{\text {a }}$ & $-130[2]$ \\
finite four-center cluster & $-124[15,16]$ & $-198[16]$ & $-131[16]$ \\
periodic & --- & $-182[19]$ & $-140[9]$ \\
\hline
\end{tabular}

(a) Differences in the reported $J$ value are related to different basis sets used in the calculations.

\section{ACKNOWLEDGMENTS}

The «Laboratoire de Chimie et Phy sique Quantiques » is « Unité Mixte de Recherche » of the CNRS (UMR5626).

\section{REFERENCES}

1. J. Miralles, J. P. Daudey, R. Caballol, Chem. Phys. Lett. 198, 555 (1992).

2. C. J. Calzado, J. Cabrero, J.P. Malrieu, R. Caballol, J. Chem. Phys. 116, 2727 (2002); ibid. 116, 3985 (2002).

3. J. Cabrero, C. J. Calzado, D. Maynau, J. P. Malrieu, R. Caballol, J. Phys. Chem. A 106, 8146 (2002).

4. C. J. Calzado, J. P. Malrieu, J. F. Sanz. J. Phys. Chem. A 102, 3659 (1998).

5. G. Aeppli, S. M. Hayden, H. A. Mook, Z. Fisk, S. W. Cheong, D. Rytz, J. P. Remeika, G. P. Espinosa, A. S. Cooper, Phys. Rev. Lett. 62, 2052 (1989).

6. R.P. Singh, P.A. Fleury, K.B. Lyons, P.E. Sulewski, Phys. Rev. Lett. 62, 2736 (1989).

7. I. de P. R. Moreira, F. Illas and R. L. Martin Phys. Rev. B 65155102 (2002) and references therein. 
8. I. de P. R. Moreira and R. Dovesi Int. J. Quantum Chem. 99, 811 (2004).

9. I. de P.R. Moreira, C.J. Calzado, J.P. Malrieu, F. Illas Phys. Rev. Lett. 97, 087003 (2006).

10. C. J. Calzado, S. Evangelisti, D. Maynau. J. Phys. Chem. A 107, 7581 (2003).

11. C. J. Calzado, J. P. Malrieu, J. Cabrero, R. Caballol. J. Phys. Chem. A 104, 11636 (2000).

12. T. P. Mitchell, W. H. Bernard, J. R. Wasson, Acta Crystallogr. Sect. B 26, 2096 (1970).

13. B. Bories, D. Maynau, M. L. Bonnet, J. Comp. Chem. 28, 632 (2007)

14. C.J. Calzado and D. Maynau, in preparation.

15. Z. Shi Tan, Y. Fujii, R. Nubada, M. Nimuriya, Y. Nakano, J. Chem. Soc. Dalton Trans. 2415 (1999).

16. C. J. Calzado, J. P. Malrieu, Phys. Rev. B 63, 214520 (2001).

17. C. J. Calzado, J. P. Malrieu, Phys. Rev. B 69, 094435 (2004).

18. R. L. Martin, F. Illas, Phys. Rev. Lett. 79, 1539 (1997).

19. I. de P.R. Moreira, C.J. Calzado, J.P. Malrieu, F. Illas, to be published 